\title{
ANIMAL HEALTH AND WELL BEING Ingestion of organic acids and cinnamaldehyde improves tissue homeostasis of piglets exposed to enterotoxic Escherichia coli (ETEC)
}

\author{
Milton J. Jiménez, ${ }^{\dagger}$ Roger Berrios, ${ }^{\ddagger}$ Sabine Stelzhammer, ${ }^{\ddagger}$ Miriam Hohmann,, \\ Waldiceu Verri Jr.," and Ana Paula F. R. L. Bracarense ${ }^{\dagger, 1}$
}

†Laboratory of Animal Pathology, Universidade Estadual de Londrina, Campus Universitário, Rodovia Celso Garcia Cid, Km 380, Londrina, Paraná 86057-970, Brazil, `BIOMIN Holding GmbH, Erber Campus 1, Getzersdorf 3131, Austria, "Laboratory of Pain, Inflammation, Neuropathy and Cancer, Universidade Estadual de Londrina, Londrina, Paraná 86057-970, Brazil

${ }^{1}$ Corresponding author: anapaula@uel.br

ORCiD number: 0000-0002-5407-2582 (A. P. F. R. L. Bracarense).

\begin{abstract}
Organic acids (OA) and phytogenic compounds have been used in pig feeding as alternatives to antibiotic growth promoters. However, few studies have evaluated the systemic effect of the combination of these additives. The aim of this study was to assess the impact of an organic acid-based feed additive (OAFA), containing a blend of OA and cinnamaldehyde, on the tissue integrity of bacterially challenged piglets. Thirty weaned piglets $21 \mathrm{~d}$ old were used in a 19-d trial. Pigs received a standard diet during the first $7 \mathrm{~d}$ and afterward were allotted to five treatments. Dietary treatments were: Control (basal diet), Escherichia coli (basal diet and challenge with E. coli), colistin (basal diet $+200 \mathrm{mg}$ colistin/ $\mathrm{kg}$ feed + challenge with E. coli), OAFA1 (basal diet + $1 \mathrm{~kg}$ OAFA/ton feed + challenge with E. coli), and OAFA2 (basal diet + $2 \mathrm{~kg}$ OAFA/ton feed + challenge with E. coli). Seven days after the beginning of the treatment, the animals were challenged with an enterotoxic strain of E. coli (K88) for pigs. Five days after the challenge, all animals were euthanized for tissue sampling for histological and oxidative stress (intestine and liver) analysis. The reduced glutathione (GSH), ferric-reducing ability potential (FRAP), and free-radical scavenging ability (ABTS) assays were used to evaluate the intestinal antioxidant defense. Lipid peroxidation and superoxide anion production were evaluated through the levels of thiobarbituric acid-reactive substances (TBARS) and nitroblue tetrazolium (NBT) reduction assay, respectively. Animals fed the OAFA (1 and 2) diets had a decrease $(P<0.05)$ on histological changes in the intestine, liver, mesenteric lymph nodes, and spleen. Greater villus height $(\mathrm{VH})$ and a higher ratio of $\mathrm{VH}$ to crypt depth (CD) were observed in animals of the OAFA2 group compared with the control and E. coli groups. The colistin and OAFA groups decreased $(P<0.05)$ the number of inflammatory cells in intestinal lamina propria. OAFA2 group increased $(P<0.05)$ intestinal cell proliferation. Colistin and OAFA2 supplementation induced a decrease $(P<0.05)$ in the levels of TBARS in both the intestine and liver compared with the E. coli group. In addition, an increase $(P<0.05)$ in GSH and FRAP ileal levels was observed in the OAFA2 group compared with E. coli group. These results show that the supplementation with OAFA in the diet of weaned piglets, especially at a dose of $2 \mathrm{~kg} /$ ton (OAFA2) protected tissues against enterotoxigenic Escherichia coli (ETEC) damage.
\end{abstract}

Key words: growth promoters, intestinal health, liver, oxidative stress, phytogenic compounds, pigs 


\begin{tabular}{ll} 
Abbreviations & \\
\hline ABTS & $\begin{array}{l}\text { free-radical scavenging ability } \\
\text { brain-heart infusion } \\
\text { crypt depth }\end{array}$ \\
CD & dimethyl sulfoxide \\
DMSO & dithiobisnitrobenzoic \\
DNTB & enterotoxigenic Escherichia coli \\
ETEC & ferric-reducing ability potential \\
FRAP & glutathione \\
GSH & malondialdehyde \\
MDA & nalidixic acid \\
Nal & nitroblue tetrazolium \\
NBT & organic acid-based feed additive \\
OAFA & optical density \\
OD & organic acids \\
OA & postweaning diarrhea \\
PWD & rifampicin \\
Rif & reactive oxygen species \\
ROS & thiobarbituric acid-reactive \\
TBARS & substances \\
& tripyridyltriazine \\
TPTZ & villus height \\
VH &
\end{tabular}

\section{Introduction}

Weaning subjects piglets to environmental, nutritional, and psychological stress, which often results in intestinal, immunological, and behavioral changes, characterized by low feed intake, growth performance, or weight loss (Pluske et al., 1997; Partanen et al., 2007). Furthermore, weaning is associated with postweaning diarrhea (PWD), which is generally caused by the proliferation of $\beta$-hemolytic strains of enterotoxigenic Escherichia coli (ETEC) (Pluske et al., 1997). A major challenge facing the swine industry today is to identify ways to control diarrhea in young pigs that will not only be profitable but also suitable for sustainable pork production. Subtherapeutic doses of antibiotics have been used in the diets of piglets to enhance the growth performance and to deal with diarrhea problems. However, the risk of antibiotic resistance development has led to the ban of diverse in-feed antibiotics. After the prohibition of antibiotic growth promoters, a diversity of dietary additives has been studied as alternatives, evaluating their potential in reducing ETEC proliferation, the incidence of postweaning diarrhea (PWD), and improvement of intestinal health and performance of weaned piglets (Halas et al., 2009). Within this group and considering their action potential, organic acids (OA) and phytogenic compounds could be relevant alternatives to develop an antibiotic-free feeding strategy (Heo et al., 2013).

OA such as formic, propionic, benzoic, fumaric, lactic, and citric acids present bacteriostatic and bactericidal properties and have been used in elevated dose in piglet diets (Suiryanrayna and Ramana, 2015). The dissociation of the OA probably leads to a reduction of the $\mathrm{pH}$ of the diet, creating a negative environment for the growth of pathogenic microorganisms and a favorable medium for the growth of acid lactic bacteria (Tsiloyiannis et al., 2001). However, the non-dissociated form of OA can penetrate the cell wall of bacteria and disrupt their normal physiology, making them effective antimicrobial agents (Papatsiros and Billinis, 2012). The addition of OA to weaned pig diets have improved growth performance (Braz et al., 2011), gastrointestinal health (Heo et al., 2013), and reduced the incidence and severity of diarrhea (Tsiloyiannis et al., 2001). It has been reported that the ingestion of OA reduces the concentration of hydroxyl radicals in the serum of weaned piglets (Long et al., 2018). Moreover, OA ameliorate intestinal morphology in weaned piglets by reducing the digesta $\mathrm{pH}$ values and maintaining the microbiota balance (Suiryanrayna and Ramana, 2015).

Plants produce a large number of secondary metabolites or phytochemicals compounds, which are important for plant homeostasis, mainly as a defense mechanism against microorganisms. Essential oils (EO), terpenoids, phenolic compounds, polypeptides, alkaloids, and polyacetylenes are the main phytochemicals present in plants (Cowan, 1999). The EO are aromatic oily liquids obtained through various processes, but the steam distillation method is the most common. Antibacterial activity of EO is mainly associated with their components such as phenols (carvacrol, eugenol, and thymol), terpenes ( $\alpha$-terpineol, terpinen-4-ol), and aldehydes, and also depending on their concentration in the oil (Burt, 2004). Cinnamaldehyde (an aromatic aldehyde and the principal constituent of cinnamon bark oil) (Putaala et al., 2017) gained renewed interest for application in pig's feed because of its antimicrobial properties (Yan and Kim, 2012). This component improves weaned piglets performance, immunity, and gut microbiota, as well as reduces the concentration of fecal noxious gas content and the incidence of diarrhea (Li et al., 2012; Yan and Kim, 2012).

The addition of a combination of $\mathrm{OA}$ and phytogenic compounds in the diet of weaned piglets possibly provides a synergistic effect, especially on productive performance (Xu et al., 2018). The present study focused mainly on intestinal health. In addition, we are interested in assessing the systemic effects of a combination of $\mathrm{OA}$ and phytogenic compounds, especially in immunologically challenged piglets. Therefore, the aim of this study was to evaluate the effects of a blend of OA and cinnamaldehyde on tissue homeostasis of piglets exposed to ETEC, as a possible alternative to preventive medication with antibiotics.

\section{Material and Methods}

The following experiment was conducted at the Universidade Estadual de Londrina (Londrina, Paraná, Brazil) swine facility. Animal use and sample collection procedures were previously approved by the Institutional Ethics Committee on the use of animals (number 13812.2017.19).

\section{Animals and experimental design}

A total of 30 piglets were used (half-castrated males and half females). The animals were weaned at $21 \mathrm{~d}$ of age, with average weights of $6.06 \mathrm{~kg}$ (SD 0.25). During $7 \mathrm{~d}$, they received a standard diet. Then, the animals were randomly divided and subjected to five treatments during $12 \mathrm{~d}$ with six repetitions per treatment. Dietary treatments were 1) Control (basal diet), 2) E. coli (basal diet and challenge with E. coli), 3) colistin (basal diet + $200 \mathrm{mg}$ colistin/kg feed + challenge with E. coli), 4) organic acid-based feed additive (OAFA) 1 (basal diet $+1 \mathrm{~kg}$ OAFA/ton feed + challenge with E. coli), and 5) OAFA2 (basal diet + $2 \mathrm{~kg}$ OAFA/ton feed + challenge with $E$. coli). The OAFA contained a minimum $18 \%$ of formic acid, $9 \%$ of acetic acid, $4.5 \%$ of propionic acid, and $3.5 \%$ flavoring substances as main ingredient cinnamaldehyde. Seven days after the beginning of the treatments, all animals (except the control group) received orally $5 \mathrm{~mL}\left(1.1 \times 10^{9} \mathrm{CFU} / \mathrm{mL}\right)$ of the enterotoxic strain of E. coli K88:LT (rNal, rRif) for swine. Five days after the challenge, all animals were euthanized (electrical stunning followed by exsanguination). Samples of jejunum, ileum, liver, mesenteric lymph node, and spleen were fixed in 
Table 1. Centesimal and nutritional composition of the experimental diets

\begin{tabular}{lcr}
\hline Ingredients (\%) & Pre-starter II & Starter I \\
\hline Corn & 39.94 & 47.70 \\
Soybean meal & 20.06 & 27.30 \\
Maxi piglets ${ }^{1}$ & 40.00 & 0.00 \\
Maxi piglets ${ }^{2}$ & 0.00 & 25.00 \\
Total & 100.00 & 100.00 \\
Nutritional levels & & \\
Protein (\%) & 19.00 & 19.00 \\
Fat (\%) & 4.59 & 3.52 \\
Calcium (\%) & 0.91 & 1.00 \\
Phosphorus available (\%) & 0.46 & 0.48 \\
Metabolizable energy (Kcal/kg) & 3403 & 3310 \\
Digestible lysine (\%) & 1.45 & 1.35 \\
Digestible methionine + cysteine (\%) & 0.81 & 0.75 \\
Digestible threonine (\%) & 0.97 & 0.90 \\
Digestible Tryptophan (\%) & 0.28 & 0.26 \\
Total Lactose (\%) & 9.85 & 5.11 \\
Mineral matter (\%) & 6.45 & 6.62 \\
\hline
\end{tabular}

${ }^{1}$ Levels of guarantee per kg of product Maxi piglets used in the pre-start ration II: Humidity (max) 100.00 g; Crude Protein (min) 154.00 g; Ethereal Extract (min) 73.00 g; Gross Fiber (max) 50.00 g; Mineral Matter (max.) 300.00 g; Calcium ( $\min / \max$ ) 16.00/24.00 g; Sodium (min) 8,000.00 mg; Phosphorus (min) 10.00 g; Lysine (min) $24.00 \mathrm{~g}$; Methionine $(\mathrm{min})$ 7,800.00 mg; Threonine $(\mathrm{min}) 15.00 \mathrm{~g}$; Tryptophan (min) 3,300.00 mg; Cobalt (min) 2.00 mg; Iron (min) $142.00 \mathrm{mg}$; Iodine (min) $2.00 \mathrm{mg}$; Manganese (min) $100.00 \mathrm{mg}$; Selenium (min) $1.00 \mathrm{mg}$; Folic Acid (min) $9.00 \mathrm{mg}$; Choline (min) 1,225.00 mg; Niacin (min) 95.00 mg; Calcium Pantothenate (min) $31.00 \mathrm{mg}$; Vitamin A (min) 32,000.00 IU; Vitamin B1 (min.) $6.00 \mathrm{mg}$ Vitamin B12 (min) 96.00 mcg; Vitamin B2 (min) 19.00 mg; Vitamin B6 (min) 9.00 mg; Vitamin D3 (min) 6,400.00 IU; Vitamin E (min) 160.00 IU; Vitamin H (min) 0.60 mg; Vitamin K (min) 6.00 mg. Main ingredients: Biscuit Bran (27\%), Whole Soybean Extract (9\%), Plasma $(5 \%)$, Bicalcic Phosphate (3\%), Calcitic calcary $(2 \%)$, Common Salt, Whey (33\%), Milk powder (4\%).

${ }^{2}$ Levels of guarantee per $\mathrm{kg}$ of product Maxi piglets used in the start ration I: Humidity (max) 100.00 g; Crude Protein (min) 84.00 g; Ethereal Extract (min) 63.00 g; Gross Fiber (max) 50.00 g; Mineral Matter (max.) 300.00 g; Calcium $(\min / \max ) 28.00$ / 44.00 g; Phosphorus (min) 15.00 g; Sodium (min) 11.00 g; Lysine (min) $20.00 \mathrm{~g}$; Methionine $(\mathrm{min})$ 4,900.00 mg; Threonine (min) $10.00 \mathrm{~g}$; Cobalt (min) $5.00 \mathrm{mg}$; Iron (min) $251.00 \mathrm{mg}$; Iodine (min) $5.00 \mathrm{mg}$; Manganese (min) 200.00 mg; Selenium (min) $2.00 \mathrm{mg}$; Folic Acid (min) $3.00 \mathrm{mg}$; Choline (min) 2,672.00 mg; Niacin (min) $129.00 \mathrm{mg}$; Calcium Pantothenate (min) 60.00 mg; Vitamin A (min) 38,000.00 IU; Vitamin B1 (min.) 8.00 mg; Vitamin B12 (min) 129.00 mcg; Vitamin B2 (min) $25.00 \mathrm{mg}$; Vitamin B6 (min) $12.00 \mathrm{mg}$; Vitamin D3 (min) 9,000.00 IU; Vitamin E (min) 807.00 IU; Vitamin H (min) 0.50 mg; Vitamin K (min) 9.00 mg. Main ingredients: Biscuit Bran (33\%), Bicalcic Phosphate (7\%), Calcitic limestone (4\%), Common Salt (2\%), Sugar (19\%), Whey (29\%).

a buffered solution of formalin $10 \%$ for histological analysis and determination of a lesional score. In addition, samples of jejunum, ileum, and liver were immediately frozen at $-80^{\circ} \mathrm{C}$ for the stress oxidative analysis.

\section{Diets}

During the trial, the piglets received two diets (Table 1), formulated according to their requirements, being represented by pre-starter II ( 28 to $38 \mathrm{~d}$ old) and starter I ( 39 to $40 \mathrm{~d}$ old). The piglets were fed ad libitum. In the colistin treatment, colistin was added to the basal diet at a dose of $200 \mathrm{mg}$ colistin/kg feed. OAFA was included in the basal diet at 1 and $2 \mathrm{~kg} /$ ton feed in OAFA1 and OAFA2 groups, respectively.
Preparation of the inoculum and inoculation

E. coli strains used in the study were derived from the bacteria bank of Universidade Estadual de Londrina. These strains were recovered from clinical cases of piglets with PWD. The experiment began with the induction of resistance in isolates of E. coli (K88) to rifampicin (Rif) and nalidixic acid (Nal). The induction of antimicrobial resistance consists of cultivating the microorganism in broth and agar in different concentrations of antibiotics. The total process to obtain clones of $E$. coli resistant took $24 \mathrm{~d}$ to complete (12 d-for each antibiotic). Four samples of enterotoxigenic E. coli (K88ab; K88; 987p; paa) resistant to Nal and Rif were cultured in brain-heart infusion (BHI) broth at $37{ }^{\circ} \mathrm{C}$ for $24 \mathrm{~h}$. The samples were individually cultured and a pool was formed prior to inoculation. An aliquot was taken to perform colony-forming units (CFU/mL) determination by serial dilution and plating on MacConkey agar ( $100 \mu \mathrm{g} / \mathrm{mL} \mathrm{Nal} /$ Rif). Seven days after the beginning of the treatment, the animals received orally $5 \mathrm{~mL}$ of ETEC inoculum $\left(1.1 \times 10^{9} \mathrm{CFU} / \mathrm{mL}\right)$. The inoculum was performed as a single administration. After inoculum, the animals received $10 \mathrm{~mL}$ of $\mathrm{CaCO}_{3}$. Both administrations were performed using a syringe attached to a polyethylene tube. $\mathrm{CaCO}_{3}$ was used to increase bacterial survival in the stomach and to provide safe transfer of the inoculum into the small intestine.

\section{Histological assessment}

Following fixation, the samples were dehydrated through graded alcohols and embedded in paraffin wax. Sections $(5 \mu \mathrm{m})$ were stained with hematoxylin and eosin for histopathological evaluation. A lesion score was designed to compare histological changes based on a previous study (Terciolo et al., 2019), considering the degree of severity (severity factor) and the extent of each lesion. For each lesion, the score of the extent was multiplied by the severity factor. For each tissue, the minimal score was 0 and the maximal lesion score was 42 for jejunum and ileum, 36 for spleen, and 33 for liver and lymph nodes. The presence of intestinal bacterial adherence in each animal was used to determine the percentage of bacterial adherence. For morphometric analysis, $\mathrm{VH}$ and crypt depth (CD) were measured randomly on 30 villi using an image analysis system (MOTIC Image Plus, Richmond, Canada). The number of lymphocytes, plasma cells, and eosinophils was counted randomly on three fields per sample at $40 \times$ magnification. The number of mitotic figures in the intestinal crypts was counted in five fields per slide using $40 \times$ magnification. Each field corresponds to a surface area of $1.5 \mathrm{~mm}^{2}$.

\section{Oxidative stress response analysis}

Determination of antioxidant capacity by the reduced glutathione (GSH), ferric-reducing ability potential (FRAP), and free-radical scavenging ability (ABTS) assays

The GSH levels were evaluated by an adapted method described previously (Sedlak and Lindsay, 1968). The assay is based on the reaction of GSH with dithiobisnitrobenzoic (DNTB) acid to form the yellow derivative thionitrobenzoic acid. The tissues were homogenized using a tissue tearor (BioSpec, São Paulo, Brazil), followed by centrifugation of the homogenates $(1,500 \times$ g, $15 \mathrm{~min}$ ). Then, $0.4 \mathrm{M}$ Tris- $\mathrm{HCl}$ ( $\mathrm{pH} 8.9$ ) and $10 \mathrm{mM}$ DNTB were added to the supernatant, and the samples were vortex-mixed. The absorbances of the samples were read at $412 \mathrm{~nm}$ (Multiskan GO Microplate Spectrophotometer, Thermo Scientific, Vantaa, Finland). A standard curve was prepared using different concentrations of GSH, and other reagents as previously 
described. The results were presented as mmols of GSH per milligrams of protein.

The frozen samples were homogenized with $1.15 \% \mathrm{KCl}$, centrifuged $\left(200 \times g, 10 \mathrm{~min}, 4^{\circ} \mathrm{C}\right)$, and the supernatants were used in ferric-reducing ability potential (FRAP) and free-radical scavenging ability (ABTS) assays as previously described (Pinho-Ribeiro et al., 2015). The FRAP method is based on the production of $\mathrm{Fe}^{2+}$ ion (ferrous form) from the reduction of the $\mathrm{Fe}^{3+}$ ion (ferric form) present in the tripyridyltriazine (TPTZ) complex by means of antioxidants present in the samples. For the FRAP assay, supernatant $(15 \mu \mathrm{L})$ was mixed with deionized water $(45 \mu \mathrm{L})$ and freshly prepared FRAP reagent $(200 \mu \mathrm{L})$ (Sigma Chemical Co., St. Louis, MO, USA). The mixture was incubated for $30 \mathrm{~min}$ at $37^{\circ} \mathrm{C}$ and the absorbance was measured at $595 \mathrm{~nm}$ (Multiskan GO, Thermo Scientific). The ABTS procedure is based on the ability of the antioxidants molecules to reduce the ABTS radical cation (ABTS+). Initially, an ABTS $^{+}$solution (Sigma-Aldrich Co., St. Louis, MO, USA) was diluted with phosphate buffer saline at $\mathrm{pH} 7.4$ to reach an absorbance of 0.80 at $730 \mathrm{~nm}$. Then, $200 \mu \mathrm{L}$ of diluted ABTS.+ solution was mixed with the supernatant $(15 \mu \mathrm{L})$, and the absorbance was measured at $730 \mathrm{~nm}$ (Multiskan GO, Thermo Scientific). The results of both assays were compared with a standard Trolox curve, prepared using different concentrations (1.5 to $30 \mu \mathrm{mol} / \mathrm{L}$, final concentrations). The protein levels in the samples were used for data normalization, and the results were expressed as nmol of Trolox (Sigma Chemical Co., St. Louis, MO, USA) equivalent per milligrams of protein in both assays.

Determination of the oxidative response by nitroblue tetrazolium (NBT) and thiobarbituric acid-reactive substances (TBARS) assays

The superoxide anion production was determined by the reduction of the redox dye NBT (Amresco, Solon, OH, USA) as described by Fattori et al. (2015). The tissues were homogenized, and NBT solution $(1 \mathrm{mg} / \mathrm{mL})$ was added to homogenates. Then, after the removal of the supernatant, the formazan precipitated was solubilized by adding $2 \mathrm{M}$ of $\mathrm{KOH}$ and dimethyl sulfoxide (DMSO). To measure the optical density (OD), a microplate spectrophotometer reader (Multiskan GO, Thermo Scientific) at $600 \mathrm{~nm}$ was used. For normalization of the data, the protein levels in the samples were used, and the results were presented as NBT reduction (OD per milligram of protein).

Lipid peroxidation in tissues was assessed by determining TBARS levels using an adapted method (Guedes et al., 2006). This procedure measures malondialdehyde (MDA) an intermediate product of lipid peroxidation. The protein content of the homogenate was removed by the addition of trichloroacetic acid $(10 \%)$ followed by centrifugation $\left(1,000 \times 9,3 \mathrm{~min}, 4^{\circ} \mathrm{C}\right)$; then, thiobarbituric acid $(0.67 \%)$ was added to precipitate. MDA was determined by the difference between absorbances at 535 and $572 \mathrm{~nm}$ using a microplate spectrophotometer reader (Multiskan GO, Thermo Scientific). The results were presented as TBARS (OD A535-A572/mg of protein).

\section{Statistical analysis}

For statistical analysis, the results were presented as mean \pm SD and analyzed for residue normality (Shapiro test) and homogeneity of variances (Bartlett's test). When both assumptions were met and validated, analysis of variance (ANOVA) was applied followed by Tukey's test for multiple comparisons, using treatment as the classification factor. For the percentage of intestinal bacteria adherence, Duncan test was used. The results were statistically analyzed considering a 5\% significance level, using the statistical software GraphPad Prism 6.01 (GraphPad Software Inc., La Jolla, CA, USA).

\section{Results}

\section{Effects of colistin and OAFA on the intestine}

In the intestine, the main findings observed in all groups were edema of the lamina propria, mild villi atrophy, and fusion and flattening of enterocytes (Figure $1 \mathrm{~A}$ and $\mathrm{B}$ ). These findings were more severe in the E. coli group with apical necrosis as the main lesion (Figure 1B). In addition, an increased frequency of bacterial adherence on enterocytes was verified in this group.

The lesion score of jejunum and ileum was shown in Figure $1 \mathrm{C}$ and D. A significant decrease ( 2.5-fold) in the lesion score was observed in the jejunum of piglets of the OAFA1 and OAFA2 groups when compared with the controls. In the jejunum, a significant reduction $(P=0.046)$ in the percentage of bacterial adherence was observed in the OAFA2 group compared with the E. coli $(0 \%$ and $66 \%$ of bacterial adherence for OAFA2 and E. coli groups, respectively) (Figure 2A and B). Additionally, a significant increase $(P=0.04)$ in villi height was observed in the jejunum of piglets fed with the OAFA2 diet compared with the E. coli group (Figure $3 \mathrm{~A})$. On the other hand, a significant decrease $(P=0.04)$ in the $C D$ of pigs receiving the OAFA2 diet was noted compared with the E. coli group (Figure 3A). Piglets of the colistin and OAFA2 groups showed a significant increase $(P=0.006$ and $P=0.001)$ in the ratio $\mathrm{VH}: \mathrm{CD}$ compared with the E. coli group (Figure $3 \mathrm{~B})$.

In the ileum, a decrease in the lesion score $(P=0.006)$ was verified in the OAFA2 group compared with the E. coli group (Figure 1D). No significant difference in the percentage of bacterial adherence was observed in the ileum among the treatments $(40 \%, 50 \%, 17 \%, 17 \%$, and $25 \%$ of bacterial adherence for control, E. coli, colistin, OAFA1, and OAFA2 groups, respectively) (Figure $2 \mathrm{~A}$ ). In addition, a significant increase $(P<0.05)$ in villi height was observed in piglets of the OAFA2 group compared with the control $(P=0.008)$, E.coli, and OAFA1 $(P=0.001)$ groups (Figure 3A). A significant decrease $(P=0.04)$ in the $\mathrm{CD}$ of pigs that received the colistin was remarked compared with the E. coli group (Figure $3 \mathrm{~A}$ ). Concerning the ratio VH:CD in the ileum, piglets fed OAFA2 and colistin showed a significant increase $(P=0.001)$ compared with the $E$. coli group (Figure $3 B)$.

A significant increase $(P<0.05)$ in the number of lymphocytes in both intestinal regions and of plasma cells in the jejunum was observed in piglets of the E. coli group when compared with the other treatments (Figure 4). In addition, in the jejunum, the number of eosinophils increased in pigs submitted to the E. coli diet in comparison with control, colistin, and OAFA2 groups. Enterocyte proliferation was estimated by counting the number of mitosis figures in the intestinal crypts. The mean numbers of mitosis per field in the jejunum were $3.50,4.53$, 4.30, 4.80, and 5.10 for the control, E. coli, colistin, OAFA1, and OAFA2 groups, respectively. A significant increase $(P<0.001)$ in cell proliferation was observed in the jejunum of the pigs of the OAFA2 group when compared with the control. In the ileum, the mean numbers of mitosis per field for the control, E. coli, colistin, OAFA1, and OAFA2 groups were 4.20, 4.57, 4.40, 4.73, and 4.93, respectively. No significant differences were observed.

\section{Effects of colistin and OAFA on the liver}

In the liver, a significant increase (3-fold, $P=0.009$ ) in the lesional score was observed in pigs receiving only the bacterial inoculum (E. coli group) compared with the other groups (Figure 5A). The main histological changes were vacuolization of hepatocytes and lymphocytic infiltrate (Figure 5B-D). 


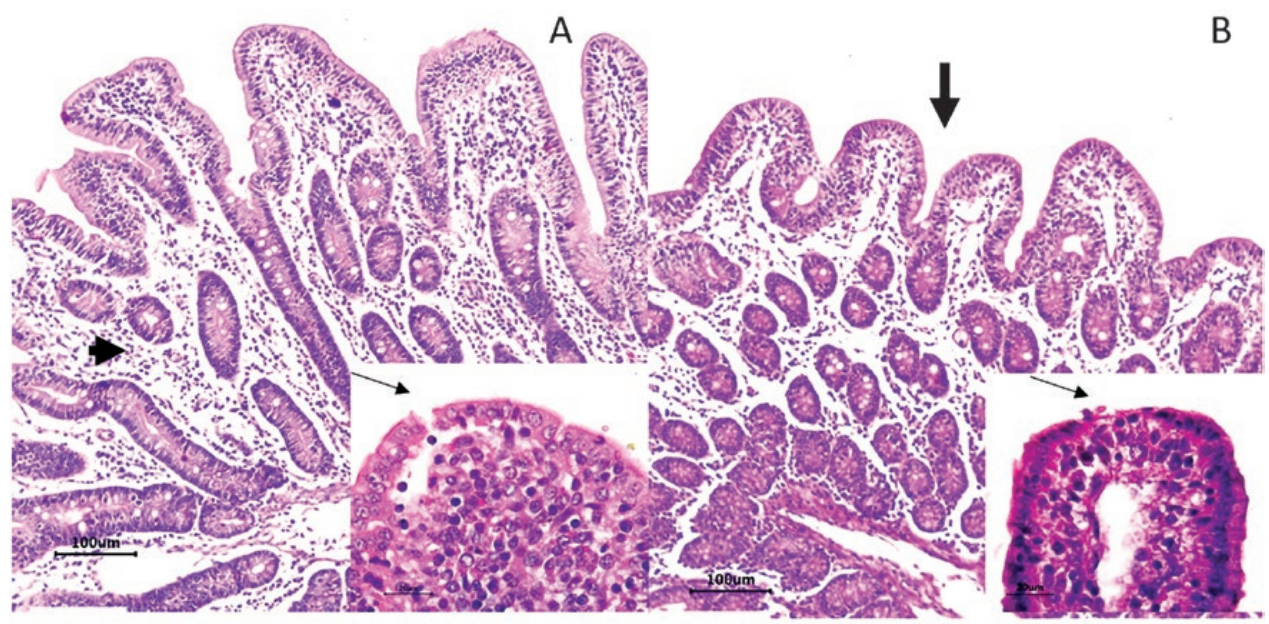

C

D
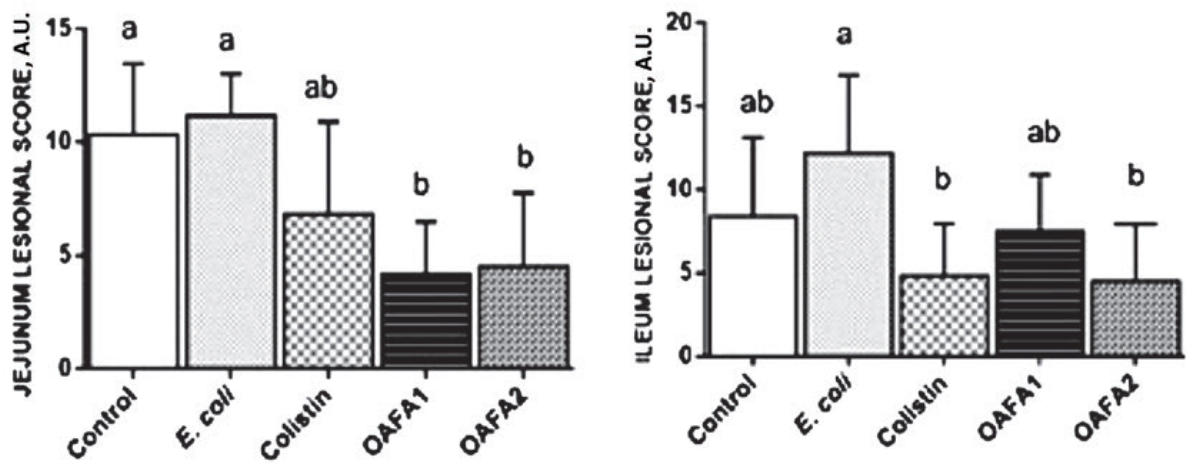

Figure 1. Jejunum of piglets. (A) Control group. Edema of the lamina propria (arrowhead) and mild villi fusion, HE, Bar $100 \mu$ m. Insert: change in enterocytes morphology from a columnar to a cubic morphology (arrow), HE, 60x; (B) E. coli group. Mild villi atrophy (arrow) and edema of lamina propria. Insert: enterocyte apical necrosis (arrow), HE, 60x; (C and D) Lesion score in the jejunum and ileum of piglets. Values are means (arbitrary units) with standard deviation ( \pm SD) represented by vertical bars $\left(n=6\right.$ animals). ${ }^{\text {a,b }}$ Mean values with unlike letters are significantly different $(P<0.05)$. OAFA, blend of organic acids and cinnamaldehyde; OAFA1, basal diet supplemented with $1 \mathrm{~kg}$ OAFA/ton feed; OAFA2, basal diet supplemented with $2 \mathrm{~kg}$ OAFA/ton feed.

A

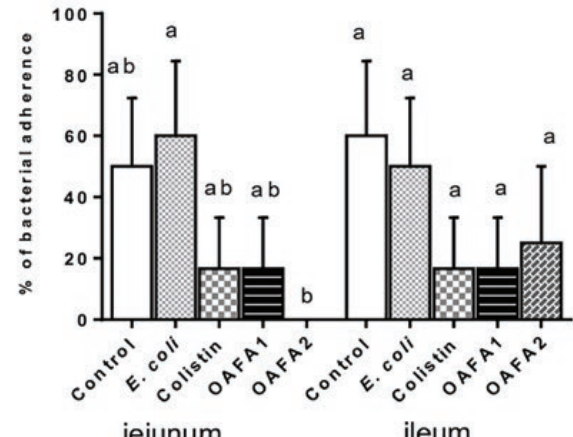

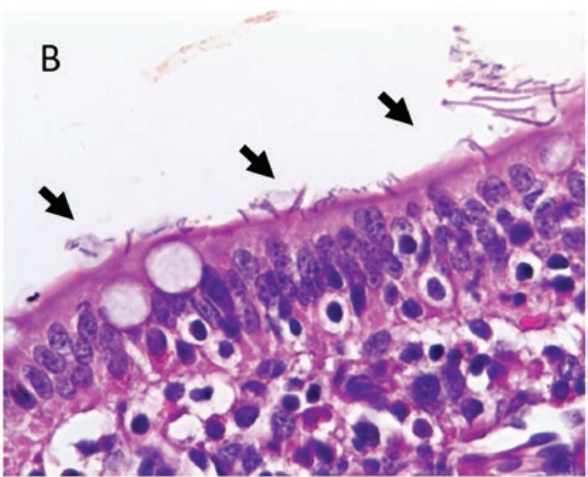

Figure 2. Effects of colistin and OAFA on piglets' intestinal bacterial adhesion. (A) Percentage of intestinal bacterial adhesion in the different treatments. Duncan test. Percentage values with unlike letters are significantly different $(P<0.05)$. (B) Jejunum of the E. coli group showing bacterial adhesion to enterocytes (arrows), HE, 60x. OAFA, blend of organic acids and cinnamaldehyde; OAFA1, basal diet supplemented with $1 \mathrm{~kg}$ OAFA/ ton feed; OAFA2, basal diet supplemented with $2 \mathrm{~kg}$ OAFA/ton feed.

Effects of colistin and OAFA on the mesenteric lymph nodes and the spleen

In the mesenteric lymph nodes, a significant decrease $(P=0.0009)$ in the lesional score was observed in the groups that received the additives compared with animals of E. coli group (Figure 6A). Changes were verified in all groups, and macrophage activation in medullary sinuses and subcapsular eosinophilic infiltrate are among the main histological changes.

In the spleen, a significant decrease in the lesion score was in the pigs that received the additive (OAFA2) when compared with the control, E. coli, and colistin groups $(P=0.001, P=0.0001$, and $P=0.03$, respectively) (Figure $6 \mathrm{~B}$ ). A significant increase $(P=0.002)$ was observed in the piglets of OAFA1 group in relation 


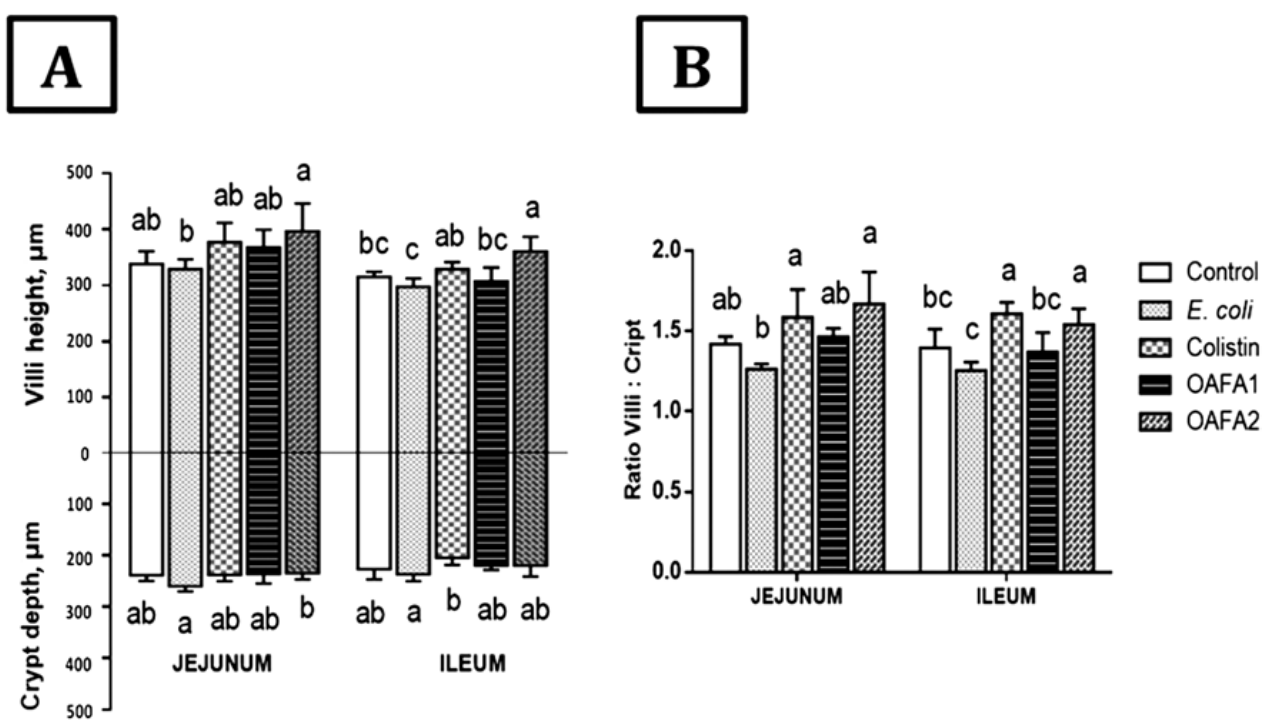

Figure 3. Effects of treatments on VH and CD in jejunum and ileum of piglets. Values are means of height and depth ( $\mu \mathrm{m})$ with their standard deviation $( \pm \mathrm{SD})$ represented by vertical bars ( $n=6$ animals). a,b Mean values with unlike letters are significantly different $(P<0.05)$. OAFA, blend of organic acids and cinnamaldehyde; OAFA1, basal diet supplemented with $1 \mathrm{~kg}$ OAFA/ton feed; OAFA2, basal diet supplemented with $2 \mathrm{~kg}$ OAFA/ton feed.
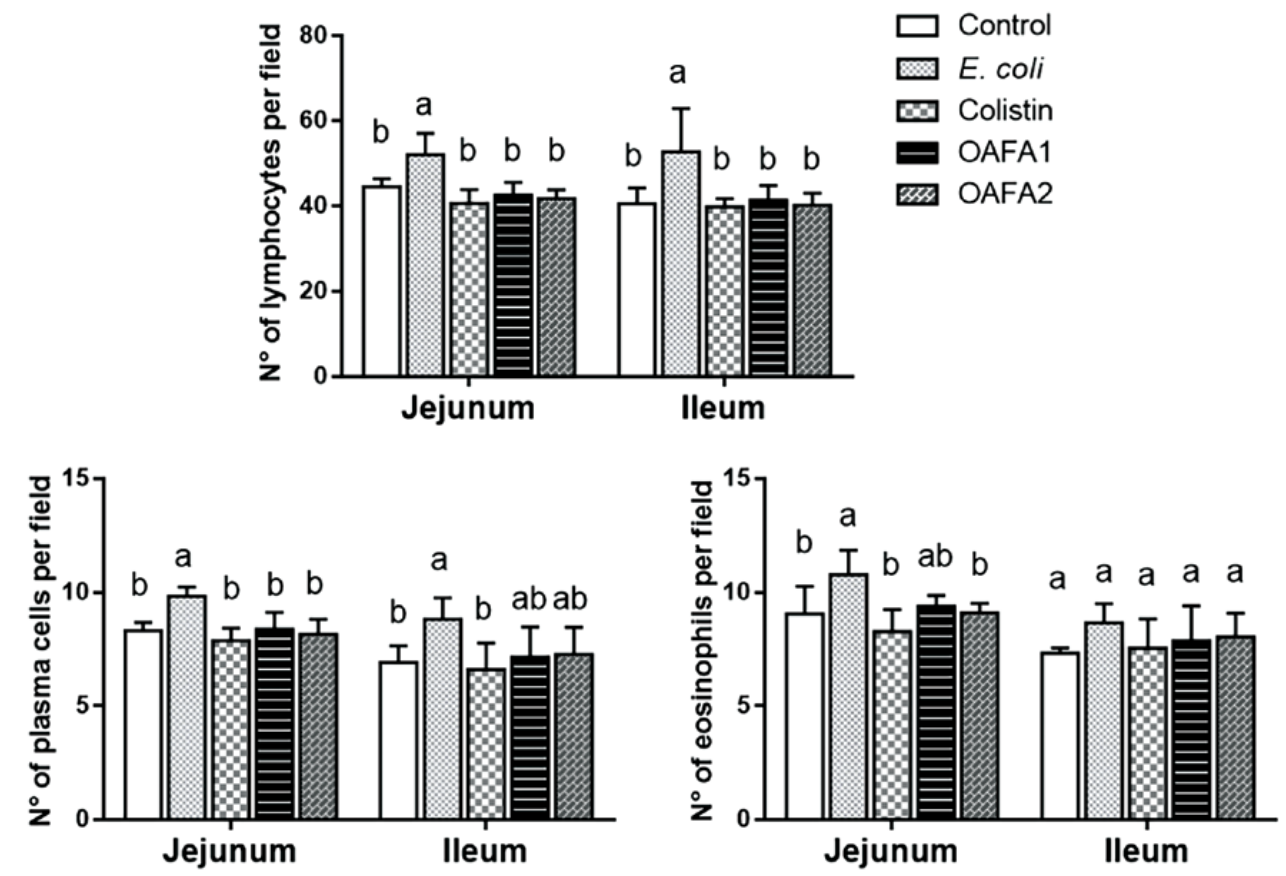

Figure 4. Effects of treatments on the number of inflammatory cells in the intestinal lamina propria of piglets. Values are mean numbers of inflammatory cells per field $\left(n=6\right.$ animals) with standard deviation $( \pm S D)$ represented by vertical bars. ${ }^{a}, \mathrm{~b}$ Mean values with unlike letters are significantly different $(P<0.05)$. OAFA, blend of organic acids and cinnamaldehyde; OAFA1, basal diet supplemented with $1 \mathrm{~kg}$ OAFA/ton feed; OAFA2, basal diet supplemented with $2 \mathrm{~kg}$ OAFA/ton feed.

to the E. coli group. Animals exposed to colistin had no effect compared with E. coli group. The main findings were hyperplasia of lymphoid follicles in both control and E. coli groups. Mild neutrophil infiltrate was observed in all groups.

Antioxidant capacity and oxidative response in the intestine of piglets fed the experimental diets

The addition of OAFA2 in challenged animals induced a significant reduction $(P=0.009$ and $P=0.01)$ in the concentration of TBARS and NBT levels (Table 2) and a significant increase
$(P=0.009$ and $P=0.002)$ in GSH and FRAP ileal levels when compared with $E$. coli group. ABTS levels showed a significant decrease $(P=0.001)$ in the jejunum of the animals of the colistin group compared with the control group, and a significant decrease $(P=0.009)$ in the ileum of the animals of the OAFA1 group, when compared with the E. coli group. On the other hand, GSH levels in the jejunum of the pigs of OAFA2 showed a significant increase $(P=0.001)$ when compared with the colistin group. In the ileum of the piglets supplemented with OAFA2, a 

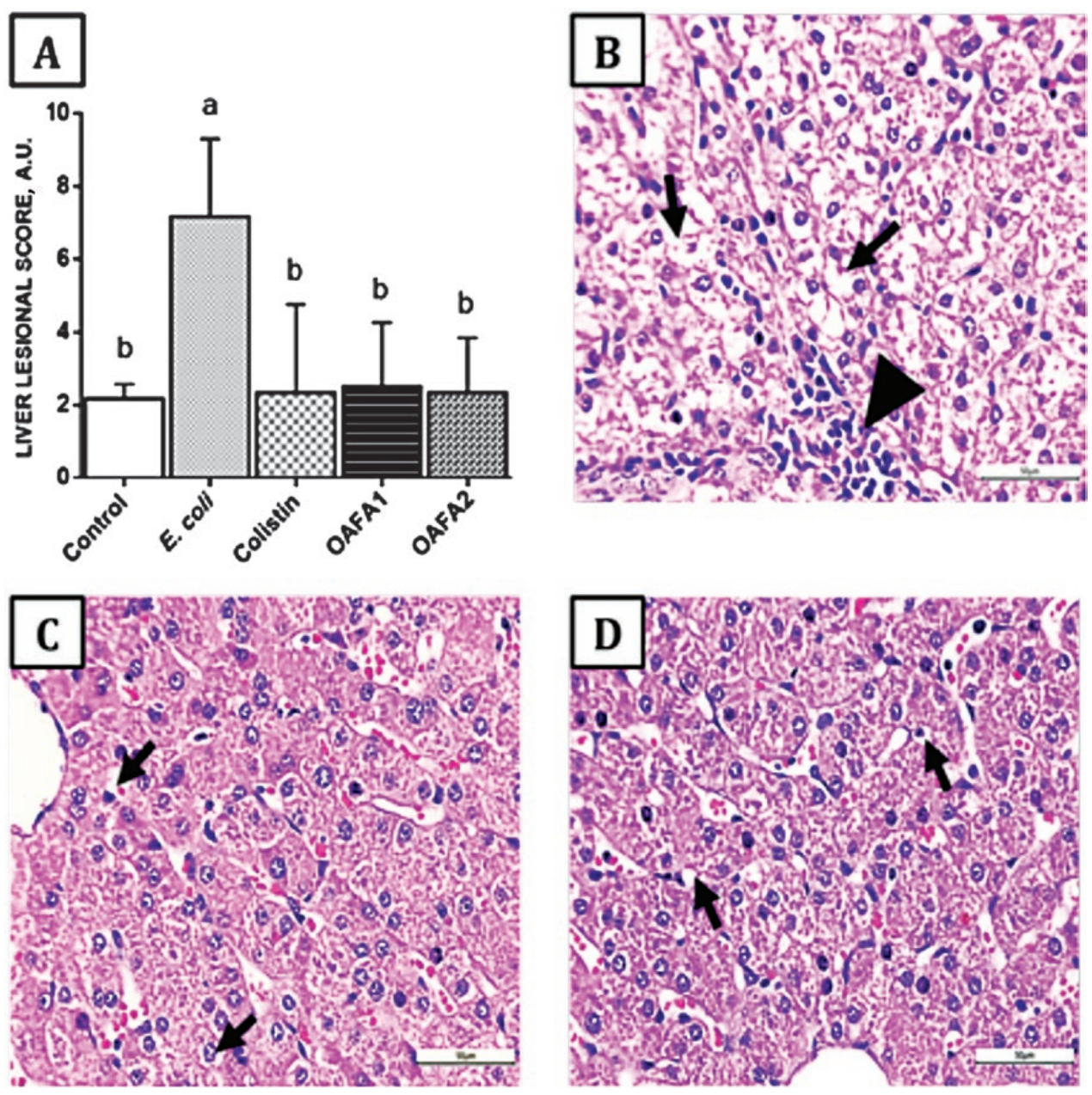

Figure 5. Effects of treatments on the liver of piglets. (A) Hepatic lesion score, values are mean \pm SD for six animals. a,b Mean values with unlike letters are significantly different by Tukey's test $(P<0.05)$. (B) E. coli group. Hepatocyte cytoplasmatic vacuolization (arrow) and lymphocytic infiltrate (arrow head), HE, 40x; (C) Colistin group. Mild hepatocyte cytoplasmatic vacuolization (arrow), HE, 40x; (D) OAFA2 group. Mild hepatocyte cytoplasmatic vacuolization (arrow), HE, 40x. OAFA, blend of organic acids and cinnamaldehyde; OAFA1, basal diet supplemented with $1 \mathrm{~kg}$ OAFA/ton feed; OAFA2, basal diet supplemented with $2 \mathrm{~kg}$ OAFA/ton feed.
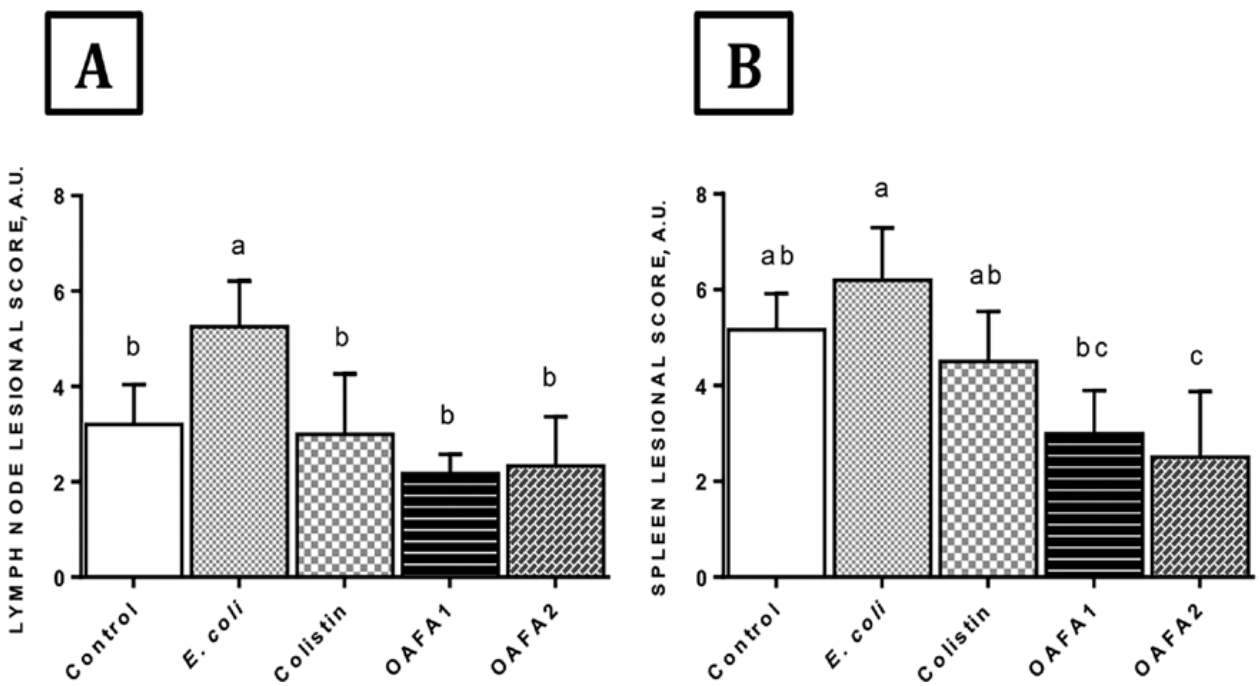

Figure 6. Effects of treatments on lymphoid organs of piglets. (A) Lymph node and (B) Spleen lesion score. Values are mean \pm SD for six animals. ${ }^{a, b}$ Mean values with unlike letters are significantly different by Tukey's test $(P<0.05)$. OAFA, blend of organic acids and cinnamaldehyde; OAFA1, basal diet supplemented with $1 \mathrm{~kg}$ OAFA/ ton feed; OAFA2, basal diet supplemented with $2 \mathrm{~kg}$ OAFA/ton feed. 


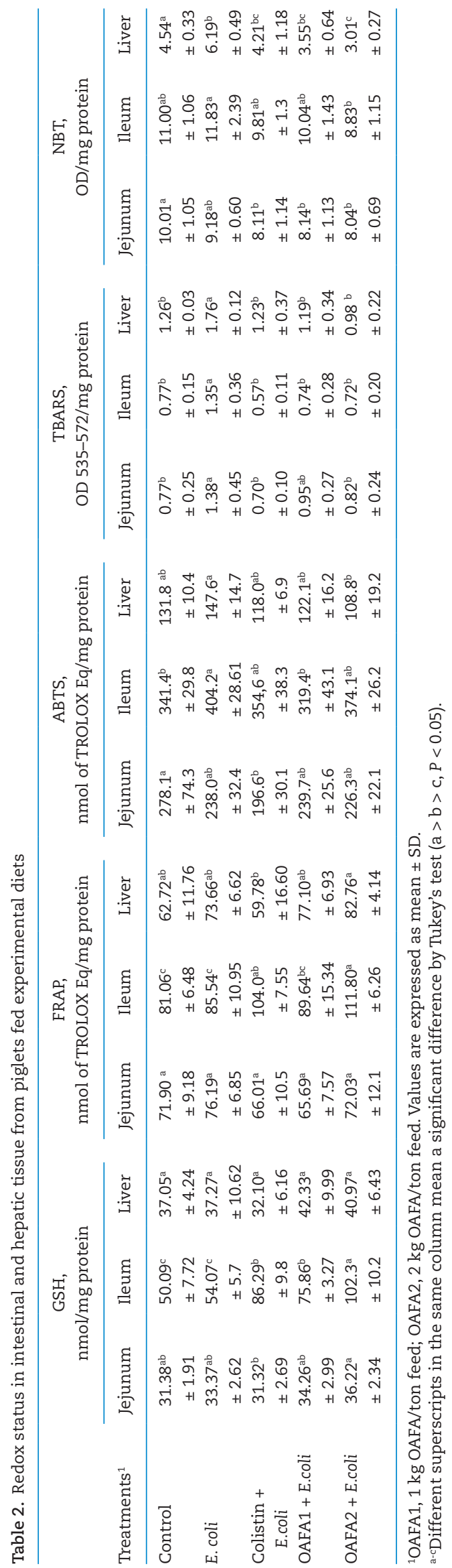


significant increase $(P=0.002)$ in GSH levels compared with the other groups was also observed (Table 2).

\section{Antioxidant capacity and oxidative stress in liver of piglets fed the experimental diets}

Significant reductions of TBARS, NBT, and ABTS $(P=0.0009$, $P=0.0001$, and $P=0.009$, respectively) were observed in the animals that received the feed additive (OAFA2) compared with the E. coli group. OAFA1 supplementation also significantly decreased the TBARS levels $(P=0.02)$ when compared with $E$. coli group. The levels of GSH remained unchanged. In addition, the liver of animals fed OAFA2 diet showed a significant increase $(P=0.009)$ of FRAP levels when compared with colistin group (Table 1). Significant increases $(P=0.035$, for both) in the levels of TBARS and NBT were observed in the animals of the $E$. coli group compared with the control group.

\section{Discussion}

There is a growing awareness about the use of feed medication (antimicrobials as growth promoters) in livestock diets and the risk of developing cross-resistance of pathogens to antibiotics, threatening human and animal health. OA, as well as phytogenic compounds, have received much attention in pig production due to their natural antimicrobial properties and beneficial effects on growth performance (Thacker, 2013). The small intestine plays an important role in the etiology of digestive disorders. The gastrointestinal tract not only works for the digestion and absorption of nutrients, but also acts as a selective barrier protection against harmful antigens, toxins, and other pathogens (König et al., 2016).

In the present study, we have investigated the effect of a mixture of $\mathrm{OA}$ and cinnamaldehyde on the intestinal integrity of weanling pigs challenged with ETEC to evaluate if they could help to improve intestinal health and thus serve as an alternative to antibiotics. OA and phytogenic compounds act through different mechanisms on the intestinal tract. OA are known to be able to prevent the colonization and proliferation of pathogenic bacteria in the intestine, exerting positive actions, such as maintaining organ integrity and promoting mucosal villus height (VH) (Heo et al., 2013). On the other hand, phytogenic compounds like cinnamaldehyde can stimulate digestive secretions modulating the microbiota and fermentation pattern of the GIT of pigs (Niu et al., 2006). The main lesions observed in our study were edema of the lamina propria, mild villi atrophy, and fusion and flattening of enterocytes in all groups. Villus atrophy is mainly caused by an increased rate of cell apoptosis and decreased rate of renewal (Pluske et al., 1997). It is important to highlight that these findings were less severe in the groups that received the OAFA when compared with the control and E. coli groups. A decrease of approximately 2.5-fold of the lesion score was observed in the OAFA groups compared with the control and E. coli groups, being very similar to the score of the colistin group.

A reduction in the percentage of intestinal bacterial adherence was observed in the jejunum of the animals receiving OAFA2 diet compared with E. coli group. To cause intestinal infection, ETEC must first adhere to the intestinal mucosa through the fimbria, increasing intestinal permeability, thus reducing the barrier function (Fairbrother et al., 2005). Our results indicate that the addition of the high level of OAFA in the diet prevented the attachment of pathogenic bacteria to the jejunal mucosa, thus minimizing its associated damage, which is consistent with the results of intestinal morphology obtained in this study. It is possible that the decrease in the number of pathogenic bacteria in the intestine enhances the proliferation of enterocytes resulting in an improvement of intestinal morphology.

An increase in ileal $\mathrm{VH}$ was observed in the intestine of the animals fed the OAFA (OAFA2) when compared with E. coli group. Similarly, when weaned piglets received a mixture of $\mathrm{OA}$ and phytogenic compounds, an increase in jejunal VH was observed (Callegari et al., 2016; Xu et al., 2018). Longer VH is considered as an indicator of an improved absorptive capacity of the small intestine and a healthy gut (Pluske et al., 1997). Data regarding the effects of OA on intestinal morphometry of weaned piglets are controversial. Previous studies observed an increase (Braz et al., 2011), a decrease (Gomes et al., 2007), and no effect (Namkung et al., 2004) in VH. These different effects are also found in weaned piglets supplemented with phytogenic compounds (Manzanilla et al., 2006; Kroismayr et al., 2008). The divergent results reported are probably related to the differences in OA and phytogenic compounds concentration and also the mixture of products used.

A decrease in CD was observed in the jejunum and ileum of OAFA groups compared with the E. coli group, remaining similar to the colistin group. Similar results were reported in previous studies using a mixture of $\mathrm{OA}$ and phytogenic compounds (Callegari et al., 2016) and herbal extracts plus OA (Namkung et al., 2004) in weaning piglets. In our study, deeper crypts were observed in the E. coli group, coinciding with shorter villi. In a previous study, piglets challenged with ETEC showed villous atrophy when weaned at 16 and $18 \mathrm{~d}$, but not at $20 \mathrm{~d}$; this change may be followed by an increase in the depth of the crypts (McLamb et al., 2013). The increase in CD is compatible with an increase in crypt-cell production rate, and an overall stimulation of cell turn-over in the small intestine that has commonly been associated with a reduced digestive and absorptive capacity (Pluske et al., 1997).

The net absorption in the small intestine depends on the $\mathrm{VH} / \mathrm{CD}$ ratio because the enterocytes show a secretory function when they are in the crypt and an absorption function when they migrate to the villi (Manzanilla et al., 2006). It is known that decreases in this ratio impair absorption and increases the diarrhea risk. Thus, the VH/CD ratio is a useful criterion for evaluating intestinal health and function (Pluske et al., 1997). An increase in $\mathrm{VH} / \mathrm{CD}$ ratio in the jejunum and ileum was observed in this study in pigs fed diets with colistin and OAFA2 when compared with the E. coli group. It is interesting to note that the $\mathrm{VH} / \mathrm{CD}$ ratio values in the OAFA2 treatment were similar to those of the colistin group. Similarly, weaned pigs receiving a blend of $\mathrm{OA}$ and phytogenic compounds showed an increase in the VH/ CD ratio in the jejunum (Callegari et al., 2016; Xu et al., 2018).

Epithelial cell proliferation was estimated by counting mitotic figures in intestinal crypts of the jejunum and ileum. We observed a significant increase in proliferating cells in the jejunum of the pigs of the OAFA2 group when compared with control and the colistin group. Ingestion of $\mathrm{OA}$, particularly butyric acid results in a positive effect on enterocyte proliferation (Bedford and Gong, 2018). Concerning phytogenic compounds, no effect in cell proliferation was observed (Putaala et al., 2017). Considering these studies, we hypothesized that the increase in cell proliferation observed in the groups supplemented with the OAFA was due mainly to the action of OA. The beneficial effect of OA on enterocyte proliferation not only could be due to their antimicrobial activity producing changes in the intestinal microbiota (Partanen et al., 2007) but also due to the fact that OA can be used as a source of energy by the epithelial cells of the 
intestine stimulating the growth of the intestinal mucosa (Heo et al., 2013).

The intestinal lamina propria is populated by resident immune cells, including plasma cells, T cells, and macrophages, which constitute over $60 \%$ of the cellular population (König et al., 2016). In this study, a greater number of lymphocytes were observed in both intestinal regions of the E. coli group compared with other groups, indicating the tissue response to the high amount of antigen in the lumen. On the other hand, the number of lymphocytes in colistin and OAFA groups remained similar, suggesting a lower need for immune activity in the gut due to the antimicrobial action of colistin, OA, and phytogenic compound.

The liver is one of the primary defense lines against microbes and toxins crossing the intestinal barrier. Although the intestine is the preferential site in which ETEC-induced toxicity develops, ETEC infections also affect liver tissue, probably associated with increased oxidative stress, resulting in structural damage and cellular injury (Deng et al., 2015). Few reports have explored the relationship between liver injury and ETEC infection (Deng et al., 2015; Gong et al., 2018). In this study, ETEC induced hepatic lesions, mainly in animals receiving no additives (E. coli group). Vacuolization of hepatocytes and lymphocytic infiltrate were the main lesions observed in the liver of pigs of all groups. Ingestion of diets containing OAFA induced a significant decrease (3-fold) in liver changes compared with $E$. coli group, indicating that it may play a positive role in protecting liver tissue in weaning pigs. A study in early-weaned pigs has provided evidence for the beneficial effects of oral supplementation of phytogenic compounds and OA on the liver (Manzanilla et al., 2006). However, until now the effects of these compounds on the liver of weaned piglets subjected to immunological challenge have not been investigated.

In the present study, mild changes were verified in the mesenteric lymph nodes of piglets of all groups, mostly macrophage activation in medullary sinuses and subcapsular eosinophilic infiltrate. These changes were more marked in the E. coli group, probably due to a rise in the bacterial translocation. Similar results were previously described (Lessard et al., 2009). When inflammation occurs, as in infections with pathogenic E. coli or after weaning, the epithelial barrier and tight junctions functionality are impaired, facilitating translocation of pathogens into the body, particularly mesenteric lymph nodes (König et al., 2016). This translocation could be, at least in part, a predisposing factor for systemic infection. In our study, diets supplemented with OAFA induced a decrease (2-fold) in lymph nodes histological changes compared with E. coli group, indicating that the ingestion of this additive could reduce bacterial translocation to mesenteric lymph nodes.

In the spleen, the main findings were hyperplasia of lymphoid follicles in the control and E. coli groups, but these findings were more evident in challenged piglets (E. coli group). Spleen injury has already been associated with $E$. coli and lipopolysaccharide challenge (Yu et al., 2017). We have verified a significant decrease in the lesion score in piglets fed the additive, especially the OAFA2 group compared with other groups, including the colistin group. Once more, these findings indicate the positive and synergistic effects of acids and cinnamaldehyde.

Oxidative stress is an important chemical mechanism that leads to biological damage, resulting from the overproduction of reactive oxygen species (ROS) and the decrease in antioxidant defense (Omonijo et al., 2017). In the present study, we observed that E. coli challenge caused intestinal and hepatic oxidative injury, indicated by an increase in superoxide anion (NBT) and lipid peroxidation (TBARS) levels. Similar results (increasedTBARS level) were reported in mice exposed to ETEC (Deng et al., 2015). In contrast, OAFA supplementation (especially OAFA2) protected the intestine and liver against $E$. coli-induced oxidative stress as demonstrated by the significant reduction in NBT and TBARS levels, as well as a slight increase in the antioxidant capacity (ABTS, FRAP, and GSH levels). OA and phytogenic compounds present systemic antioxidant properties, by reducing ROS and TBARS concentrations and increasing antioxidant enzymes (Long et al., 2018; Xu et al., 2018). Considering these data and our results, we hypothesized that the inclusion of a combination of these additives in the diet protected the piglets against hepatic oxidative damage induced by the bacterial challenge, mainly through the scavenging of ROS.

The results of the current study showed that the supplementation with OAFA induced a tissue-protective effect against ETEC damage, even in the same or better way than the antibiotic colistin. Thereby, the research provides a theoretical basis to support the use of combinations of OA and cinnamaldehyde in the swine industry, not only as enhancers of intestinal health but also beneficial to general health. Our study also suggests that this blend could be used as an alternative to antibiotics for weaning pigs.

\section{Conflict of interest statement}

None declared.

\section{Literature cited}

Bedford, A., and J. Gong. 2018. Implications of butyrate and its derivatives for gut health and animal production. Anim. Nutr. 4:151-159. doi:10.1016/j.aninu.2017.08.010

Braz, D. B., L. B. Costa, B. Berenchtein, M. L. P. Tse, V. V. Almeida, and V. S. Miyada. 2011. Acidifiers as alternatives to antimicrobial growth promoter of weanling pigs. Arch. Zootec. 60:745-756. doi:10.21071/az.v60i231.4531

Burt, S. 2004. Essential oils: their antibacterial properties and potential applications in foods-a review. Int. J. Food Microbiol. 94:223-253. doi:10.1016/j.ijfoodmicro.2004.03.022

Callegari, M. A.,A. K. Novais, E. R. Oliveira, C. P. Dias, D. L. Schmoller, M. Pereira Junior, J. Gabriela, N. Dário, J. B. Alves, and C. A. Silva. 2016. Microencapsulated acids associated with essential oils and acid salts for piglets in the nursery phase. Semina Ci. Agr. 37:2193-2208. doi:10.5433/1679-0359.2016v37n4p2193

Cowan, M. M. 1999. Plant products as antimicrobial agents. Clin. Microbiol. Rev. 12:564-582.

Deng, Y., X. Han, S. Tang, W. Xiao, Z. Tan, C. Zhou, M. Wang, and J. Kang. 2015. Magnolol and honokiol regulate the calcium-activated potassium channels signaling pathway in Enterotoxigenic Escherichia coli-induced diarrhea mice. Eur. J. Pharmacol. 755:66-73. doi:10.1016/j.ejphar.2015.03.002

Fairbrother, J. M., E. Nadeau, and C. L. Gyles. 2005. Escherichia coli in postweaning diarrhea in pigs: an update on bacterial types, pathogenesis, and prevention strategies. Anim. Health Res. Rev. 6:17-39. doi:10.1079/ahr2005105

Fattori, V., F. A. Pinho-Ribeiro, S. M. Borghi, J. C. Alves-Filho, T. M. Cunha, F. Q. Cunha, R. Casagrande, and W. A. Verri, Jr. 2015. Curcumin inhibits superoxide anion-induced painlike behavior and leukocyte recruitment by increasing Nrf2 expression and reducing NF- $\mathrm{\kappa B}$ activation. Inflamm. Res. 64:993-1003. doi:10.1007/s00011-015-0885-y

Gomes, F. E., D. O. Fontes, E. O. S. Saliba, W. M. Ferreira, E. T. Fialho, F. C. O. Silva, M. A. Silva, G. S. S. Corrêa, and G. M. Salum. 2007. Fumaric, butyric and formic acid combinations in feed formulation for recently weaned piglets. Arq. Bras. Med. Vet. Zootec. 59:1270-77. doi:10.1590/S0102-09352007000500026 
Gong, Z., Q. Liu, L. Lin, Y. Deng, S. Cai, Z. Liu, S. Zhang, W. Xiao, S. Xiong, and D. Chen. 2018. l-Theanine prevents ETECinduced liver damage by reducing intrinsic apoptotic response and inhibiting ERK1/2 and JNK1/2 signaling pathways. Eur. J. Pharmacol. 818:184-190. doi:10.1016/j.ejphar.2017.10.050

Guedes, R. P., L. D. Bosco, C. M. Teixeira, A. S. Araújo, S. Llesuy, A. Belló-Klein, M. F. Ribeiro, and W. A. Partata. 2006. Neuropathic pain modifies antioxidant activity in rat spinal cord. Neurochem. Res. 31:603-609. doi:10.1007/ s11064-006-9058-2

Halas, D., C. F. Hansen, D. J. Hampson, B. P. Mullan, R. H. Wilson, and J. R. Pluske. 2009. Effect of dietary supplementation with inulin and/or benzoic acid on the incidence and severity of post-weaning diarrhoea in weaner pigs after experimental challenge with enterotoxigenic Escherichia coli. Arch. Anim. Nutr. 63:267-280. doi:10.1080/17450390903020414

Heo, J. M., F. O. Opapeju, J. R. Pluske, J. C. Kim, D. J. Hampson, and C. M. Nyachoti. 2013. Gastrointestinal health and function in weaned pigs: a review of feeding strategies to control postweaning diarrhoea without using in-feed antimicrobial compounds. J. Anim. Physiol. Anim. Nutr. (Berl). 97:207-237. doi:10.1111/j.1439-0396.2012.01284.x

König, J., J. Wells, P. D. Cani, C. L. García-Ródenas, T. MacDonald, A. Mercenier, J. Whyte, F. Troost, and R. J. Brummer. 2016. Human intestinal barrier function in health and disease. Clin. Transl. Gastroenterol. 7:e196. doi:10.1038/ctg.2016.54

Kroismayr, A., J. Sehm, M.W. Pfaffl, K. Schedle, and W. Windisch. 2008. Effects of avilamycin and essential oils on mRNA expression of apoptotic and inflammatory markers and gut morphology of piglets. Czech J. Anim. Sci. 53:377-387. doi:10.17221/338-CJAS

Lessard, M., M. Dupuis, N. Gagnon, E. Nadeau, J. J. Matte, J. Goulet, and J. M. Fairbrother. 2009. Administration of Pediococcus acidilactici or Saccharomyces cerevisiae boulardii modulates development of porcine mucosal immunity and reduces intestinal bacterial translocation after Escherichia coli challenge. J. Anim. Sci. 87:922-934. doi:10.2527/jas.2008-0919

Li, S.Y., Y. J. Ru, M. Liu, B. Xu, A. Péron, and X. G. Shi. 2012. The effect of essential oils on performance, immunity and gut microbial population in weaner pigs. Livest. Sci. 145:119-123. doi:10.1016/j.livsci.2012.01.005

Long, S. F., Y. T. Xu, L. Pan, Q. Q. Wang, C. L. Wang, J. Y. Wu, Y. Y. Wu, Y. M. Han, C. H. Yun, and X. S. Piao. 2018. Mixed organic acids as antibiotic substitutes improve performance, serum immunity, intestinal morphology and microbiota for weaned piglets. Anim. Feed Sci. Technol. 235:23-32. doi:10.1016/j. anifeedsci.2017.08.018

Manzanilla, E. G., M. Nofrarías, M. Anguita, M. Castillo, J. F. Perez, S. M. Martín-Orúe, C. Kamel, and J. Gasa. 2006. Effects of butyrate, avilamycin, and a plant extract combination on the intestinal equilibrium of early-weaned pigs. J. Anim. Sci. 84:2743-51. doi:10.2527/jas.2005-509

McLamb, B. L., A. J. Gibson, E. L. Overman, C. Stahl, and A. J. Moeser. 2013. Early weaning stress in pigs impairs innate mucosal immune responses to enterotoxigenic E. coli challenge and exacerbates intestinal injury and clinical disease. Plos One 8:e59838. doi:10.1371/journal.pone.0059838

Namkung, H., J. Li, M. Gong, H. Yu, M. Cottrill, and C. F. M. de Lange. 2004. Impact of feeding blends of organic acids and herbal extracts on growth performance, gut microbiota and digestive function in newly weaned pigs. Can. J. Anim. Sci. 84:697-704. doi:10.4141/A04-005
Niu, C., S. Afre, and E. S. Gilbert. 2006. Subinhibitory concentrations of cinnamaldehyde interfere with quorum sensing. Lett. Appl. Microbiol. 43:489-494. doi:10.1111/j.1472-765X.2006.02001

Omonijo, F. A., L. Ni, J. Gong, Q. Wang, L. Lahaye, and C. Yang. 2017. Essential oils as alternatives to antibiotics in swine production. Anim. Nutr. 2:126-136. doi:10.1016/j. aninu.2017.09.001

Papatsiros, V., V. G. Papatsiros, and C. Billinis. 2012. The prophylactic use of acidifiers as antibacterial agents in swine. In: Bobbarala, V., editor Antimicrobial agents. London (UK): IntechOpen; p. 295-310. doi:10.5772/32278

Partanen, K., H. Siljander-Rasi, J. Pentikäinen, S. Pelkonen, and M. Fossi. 2007. Effects of weaning age and formic acid-based feed additives on pigs from weaning to slaughter. Arch. Anim. Nutr. 61:336-356. doi:10.1080/17450390701556866

Pinho-Ribeiro, F. A., M. S. Hohmann, S. M. Borghi, A. C. Zarpelon, C. F. Guazelli, M. F. Manchope, R. Casagrande, and W. A. Verri, Jr. 2015. Protective effects of the flavonoid hesperidin methyl chalcone in inflammation and pain in mice: role of TRPV1, oxidative stress, cytokines and NF-кB. Chem. Biol. Interact. 228:88-99. doi:10.1016/j.cbi.2015.01.011

Pluske, J. R., D. J. Hampson, and I. H. Williams.1997. Factors influencing the structure and function of the small intestine in the weaned pig: a review. Livest. Prod. Sci. 51:215-236. doi:10.1016/S0301-6226(97)00057-2

Putaala, H., P. Nurminen, and K. Tiihonen. 2017. Effects of cinnamaldehyde and thymol on cytotoxicity, tight junction barrier resistance, and cyclooxygenase- 1 and -2 expression in Caco-2 cells. J. Anim. Feed Sci. 26:274-284. doi:10.22358/ jafs/77058/2017

Sedlak, J., and R. H. Lindsay. 1968. Estimation of total, protein-bound, and nonprotein sulfhydryl groups in tissue with Ellman's reagent. Anal. Biochem. 25:192-205. doi:10.1016/0003-2697(68)90092-4

Suiryanrayna, M. V., and J. V. Ramana. 2015. A review of the effects of dietary organic acids fed to swine. J. Anim. Sci. Biotechnol. 6:45. doi:10.1186/s40104-015-0042-z

Terciolo C., A. P. Bracarense, P. C. M. C. Souto, A. M. Cossalter, L. Dopavogui, N. Loiseau, C. A. F. Oliveira, P. Pinton, and I. P. Oswald. 2019. Fumonisins at doses below EU regulatory limits induce histological alterations in piglets. Toxins 11:E548. doi:10.3390/toxins11090548

Thacker, P. A. 2013. Alternatives to antibiotics as growth promoters for use in swine production: a review. J. Anim. Sci. Biotechnol. 4:35. doi:10.1186/2049-1891-4-35

Tsiloyiannis, V. K., S. C. Kyriakis, J. Vlemmas, and K. Sarris. 2001. The effect of organic acids on the control of porcine postweaning diarrhoea. Res. Vet. Sci. 70:287-293. doi:10.1053/ rvsc. 2001.0476

Xu, Y. T., L. Liu, S. F. Long, L. Pan, and X. S. Piao. 2018. Effect of organic acids and essential oils on performance, intestinal health and digestive enzyme activities of weaned pigs. Anim. Feed Sci. Technol. 235:110-119. doi:10.1016/j.anifeedsci.2017.10.012

Yan, L., and I. H. Kim. 2012. Effect of eugenol and cinnamaldehyde on the growth performance, nutrient digestibility, blood characteristics, fecal microbial shedding and fecal noxious gas content in growing pigs. Asian-Australas. J. Anim. Sci. 25:1178-1183. doi:10.5713/ajas.2012.12111

Yu, J., Y. Zhang, X. Song, Y. Yang, R. Jia, X. Chen, K. Sun, L. Li, X. Zhao, Q. Cui, et al. 2017. Effect of modified pulsatilla powder on enterotoxigenic Escherichia coli O101-induced diarrhea in mice. Evid. Based Complement. Alternat. Med. 2017:3687486. doi:10.1155/2017/3687486 